\title{
Labyrinthe
}

39 | 2012 (2)

"Et si... ? " La cause du contrefactuel

\section{Des causes historiques aux possibles du passé ? Imputation causale et raisonnement contrefactuel en histoire}

Quentin Deluermoz et Pierre Singaravélou

\section{(2) OpenEdition \\ Journals}

Édition électronique

URL : http://journals.openedition.org/labyrinthe/4269

DOI : $10.4000 /$ labyrinthe.4269

ISSN : 1950-6031

Éditeur

Hermann

Édition imprimée

Date de publication : 31 décembre 2012

Pagination : 55-79

ISBN : 9782705682637

\section{Référence électronique}

Quentin Deluermoz et Pierre Singaravélou, « Des causes historiques aux possibles du passé ?

Imputation causale et raisonnement contrefactuel en histoire », Labyrinthe [En ligne], 39 | 2012 (2), mis en ligne le 10 janvier 2013, consulté le 30 avril 2019. URL : http://journals.openedition.org/

labyrinthe/4269; DOI : 10.4000/labyrinthe.4269 


\section{Des causes historiques aux possibles du passé ? Imputation causale et raisonnement contrefactuel en histoire}

Quentin Deluermoz

Pierre SingaravéLOU

La démarche contrefactuelle en histoire semble ambivalente : omniprésente dans les ouvrages historiques, son usage explicite induit de nombreux risques mais permet aux chercheurs de revisiter des questions fondamentales, telles, entre autres, celles de la causalité et du déterminisme ${ }^{1}$. L'approche contrefactuelle n'est en effet pas qu'affaire d'imagination. Aussi étrange que cela puisse paraître, elle est tout autant liée à la dimension plus scientifique de l'histoire, par son rôle dans la formulation des hypothèses et dans la recherche des causalités. La question de la causalité ou de l'imputation causale en histoire est un vaste problème qui a préoccupé aussi bien les philosophes de l'histoire que les épistémologues. Bien que les historiens qui se sont vraiment penchés sur cette question soient rares $^{2}$, la «reconstitution des causalités» est bien l'une des formes d'intelligibilité du passé la plus utilisée, que ce soit explicitement ou implicitement, consciemment ou inconsciemment.

Quotidiennement, l'historien consacre en effet une grande partie de ses efforts à identifier des «causes» et à les hiérarchiser. Les chercheurs ont parfois pu être abusés par des raisonnements dits post hoc et cum hoc, sophismes qui voient dans un phénomène la cause alors qu'il ne

\footnotetext{
1. Sur cette démarche, son histoire, ses risques, la nature des problèmes posés et, de ce fait, son possible intérêt pour l'analyse historique, nous nous permettons de renvoyer à Quentin Deluermoz et Pierre Singaravélou, «Explorer le champ des possibles. Approches contrefactuelles et futurs non advenus en histoire», Revue d'histoire moderne et contemporaine, 59/3 (2012), p. 118-143.

2. Préférant la description de la complexité des situations historiques à de complexes spéculations, les historiens français ont tendance à se méfier des explications causales. Contrairement aux chercheurs anglophones qui, notamment sous l'influence de la philosophie analytique, ont été plus enclins à spéculer sur des chaînes causales alternatives et, donc, à promouvoir l'histoire contrefactuelle.
} 
s'agit que de l'antécédent ou du corollaire ${ }^{3}$. Aujourd'hui, toutefois, les historiens se défient davantage de cette forme de causalité linéaire et ont abandonné l'explication monocausale (du type A donne B), au profit de l'analyse de la pluralité des causes d'un phénomène historique. De même, la définition positiviste selon laquelle l'analyse scientifique peut entièrement saisir la raison d'être des faits historiques ${ }^{4}$ a fait place à partir du début du $\mathrm{Xx}^{\mathrm{e}}$ siècle à une position constructiviste qui conçoit plutôt la causalité comme la construction d'un lien entre deux éléments, en l'occurrence entre deux faits historiques.

Dans tous les cas, cependant, l'interrogation contrefactuelle semble omniprésente dans les théories de la causalité : elle paraît inévitable lorsque l'on entend penser les relations entre des éléments dans le temps (faits, actes, pensées, phénomènes, dynamiques...). En tant que principe, elle s'avère ainsi indéterminée, ce qui explique qu'elle se situe aussi au cœur de la crise du causalisme. En ce sens, l'analyse contrefactuelle est à la fois un révélateur et un outil qui peut être diversement utilisé, de façon implicite ou affichée, simpliste ou élaborée, mais qui peut aussi ouvrir sur de nouvelles conceptions de la causalité.

\section{La causalité en histoire : quelques jalons}

Nous nous contentons ici de faire état de quelques remarques sur un problème délicat qui a suscité nombre de développements, tout en demeurant souvent occulté par les historiens dans leur travail quotidien. Les chercheurs font tous face à un même problème. Ils analysent un fait qui, pour reprendre les termes de Raymond Aron, «a toujours une multiplicité d'antécédents ${ }^{5} »$. Le plus souvent, l'historien doit bien se demander «comment déterminer la cause véritable». En 1952, par exemple, Patrick Gardiner s'interrogeait sur le lien entre l'assassinat de l'archiduc François-Ferdinand et la Première Guerre mondiale, sur

\footnotetext{
3. Post hoc ergo propter hoc: à la suite de cela, donc à cause de cela / Cum hoc ergo propter hoc: avec ceci, donc à cause de ceci. La succession ou la contiguïté de deux phénomènes dans l'espace et la concomitance dans le temps n'impliquent pas nécessairement de relation causale.

4. Cette conception positiviste n'est d'ailleurs déterministe que lorsque la liaison de la cause à effet est nécessaire et inévitable.

5. Raymond Aron, Introduction à la philosophie de l'histoire. Essai sur les limites de l'objectivité historique, Paris, Gallimard, 1986, p. 199.
} 
celui entre la Première Guerre mondiale et l'essor du communisme, ou plus largement sur la connexion entre l'essor du protestantisme et le développement du capitalisme, entre les écrits de Rousseau et le gouvernement de Robespierre, etc. ${ }^{6}$ Ces différents exemples posent la question de la terminologie : qu'appelle-t-on un fait historique, un événement, un effet, etc.? D’autre part, existe-t-il un véritable lien de causalité entre ces différents éléments?

Le problème est ancien. L'histoire comme discipline s'est rapidement définie comme l'étude des causes des événements passés. Ainsi Polybe, au $\mathrm{II}^{\mathrm{e}}$ siècle avant notre ère, tenta de mettre en œuvre cette recherche des causes à propos des Guerres puniques, et en profita pour concevoir une théorie des causes particulières et générales. Selon lui, la véritable cause réside dans les dispositions morales et les intentions des acteurs euxmêmes ${ }^{7}$. Mais il prend également en considération une cause structurelle qui explique l'affrontement entre les Romains et les Carthaginois : la constitution de Rome ${ }^{8}$. Suivant ce modèle énoncé par Aristote ${ }^{9}$ et Polybe, les historiens ont tendance à distinguer différents types de causalités, conjoncturelle et structurelle, particulière et générale; différentes causalités qui renvoient à l'emboîtement de différentes temporalités. Les historiens considèrent que l'identification des causes dites profondes est l'exercice le plus périlleux, mais qu'il est nécessaire car ces causes structurelles sont souvent considérées comme les plus déterminantes. Cette recherche causale induit donc une hiérarchisation des causes, qui semble être une spécificité du métier d'historien par rapport aux scientifiques.

Une fois acquise l'idée d'une pluralité de causes, une série de tensions demeurent. L'une d'elles oppose déterminisme et contingence ou, plus exactement, pose la question du continuum de l'un à l'autre. Jusqu'au XIX ${ }^{\mathrm{e}}$ siècle, les historiens ont certes fondé leur récit sur des lois de l'histoire. Mais, bien souvent, ces lois représentent des conventions qui fournissent un cadre pratique dans lequel s'insèrent des formes de logique causale moins déterministes. C'est le cas chez Polybe, qui, au sein du

6. Patrick Gardiner, The Nature of Historical Explanation, Oxford, Oxford University Press, 1952.

7. Polybe, Histoires, III, 6; voir Paul Pédech, La méthode historique de Polybe, Paris, Les Belles Lettres, 1964.

8. Ainsi, au moins depuis Polybe, c'est la détermination des causes qui confère à un fait historique sa véritable signification.

9. Dans la Physique (II, 3), le Stagirite distingue quatre types de cause : la cause matérielle, la cause formelle, la cause efficiente et la cause finale. 
cadre de la loi historique de la naissance, de la vie et de la mort des constitutions, prend en considération la psychologie des dirigeants politiques. Les philosophes et historiens de la Renaissance et des Lumières s'inscrivent aussi dans ce système déterministe de lois de l'histoire, en s'inspirant pour leur part du modèle des sciences de la nature. Ainsi, de Bodin jusqu'à Montesquieu, l'un des grands principes de justification est d'ordre mésologique : l'environnement détermine l'évolution historique $^{10}$. La notion de contingence connaît une explicitation plus ferme et argumentée au XIX ${ }^{\mathrm{e}}$ siècle. Dans ses Considérations sur la marche des idées et des événements publiées en 1872, Antoine Cournot affirme que l'histoire est une «modalité particulière de liaison entre les phénomènes qui mêlent ordre et hasard». En effet, l'histoire ne peut pas être une série d'événements qui dériveraient «nécessairement et régulièrement les uns des autres, en vertu (de) lois constantes ». Sinon, le futur serait prévisible. L'histoire ne saurait pas davantage être «une suite d'événements qui seraient sans aucune liaison entre eux » comme la suite des résultats d'une loterie qui ne peuvent constituer un récit historique. L'histoire, pour Cournot, se situe entre ces deux formes, entre nécessité et contingence. Plus précisément, le hasard n'est plus la forme que prend notre ignorance des causes; il devient la rencontre, l'interférence imprévisible de « deux séries causales indépendantes » qui s'articulent selon un processus partiellement ou totalement aléatoire. Ainsi apparaît l'idée de causalité contingente : dans ce système causal complexe, une cause accidentelle, une cause mineure peut déterminer des relations en chaînes modifiant profondément l'ensemble ${ }^{11}$.

Cette tension entre déterminisme et contingence réapparaît périodiquement, comme c'est le cas quelques années plus tard dans la controverse entre l'école méthodique et l'école durkheimienne au début du $\mathrm{Xx}^{\mathrm{e}}$ siècle. Les historiens Charles-Victor Langlois et Charles Seignobos défendent l'histoire comme étude du contingent, du singulier en opposition aux sciences de la nature qui cherchent avant tout à établir des lois. De son

10. Comme le rappelle François Dosse, L'histoire (Paris, Armand Colin, 2000), le déterminisme de Bodin annonce celui de Montesquieu. Voir Jean Bodin, Méthode pour une facile compréhension de l'histoire, dans Euvres philosophiques (1566), Paris, PUF, 1965 ; Montesquieu, De l'Esprit des lois (1748), XIX, 4, Paris, Les Belles lettres, 1961.

11. Les philosophes de l'histoire ont retenu ces éléments comme les prémisses d'une «épistémologie du probable»: Thierry Martin, «La philosophie de l'histoire de Cournot», Revue d'histoire des sciences humaines, 12/1 (2005), p. 141-162. 
côté, le sociologue durkheimien François Simiand, dans son article polémique «Méthode historique et sciences sociales » paru dans la Revue de synthèse historique en 1903, s'attaque aux historiens français de l'école méthodique car, selon lui, ils s'attachent à la description de faits contingents en s'interdisant de comprendre les véritables causes. Simiand tente alors de promouvoir la «recherche causale» qui va fortement influencer une partie de l'École des Annales.

Une tension d'un autre ordre entre continuité et rupture en histoire structure le discours historique. L'écriture de l'histoire est dominée jusqu'à la Seconde Guerre mondiale par la forme du récit linéaire, fondée sur le présupposé de la continuité entre les faits historiques. L'une des premières mises en cause volontaires de ce schéma est celle proposée par Fernand Braudel : elle mobilise l'ensemble des sciences humaines - la sociologie, la géographie, l'économie, l'anthropologie - qui, contrairement à l'histoire, se focalisent sur les continuités plutôt que le changement ${ }^{12}$. Braudel questionne l'idée de succession linéaire des faits historiques en mettant au jour différents rythmes de temporalité, temps court, temps moyen, temps long. Mais, par là même, il s'interdit d'analyser le changement et de repenser l'événement. Une autre brèche dans l'histoire linéaire est ouverte quelques années plus tard par Michel Foucault et son éloge de la discontinuité en histoire ${ }^{13}$. Foucault entend pour sa part rompre avec l'histoire des idées qui prévaut alors : une sous-discipline obsédée par l'analyse des continuités et de la diffusion des idées par cercles concentriques autour des hommes et des œuvres. L'Archéologie du savoir cherche au contraire à penser le changement, la transformation, au travers des coupures, des failles qui induisent des reconfigurations brutales de nouveaux discours et de nouveaux rôles sociaux. Cette analyse des discontinuités autorise à penser la nouveauté. Elle permet également d'appréhender de nouveaux objets, tels que l'histoire des émotions, des sensibilités, ou plus largement des représentations. Ces processus n'ont pas de linéarité et donc ne relèvent pas d'une logique causale mécaniste. Les historiens peuvent dès lors identifier des phénomènes d'émergence

12. Fernand Braudel, La Méditerranée et le monde méditerranéen à l'époque de Philippe II, Paris, Armand Colin, 1949; id., «Histoire et sciences sociales : la longue durée», Annales. Économies, sociétés, civilisations, 13/4 (1958), p. 725-753.

13. Michel Foucault, L'Archéologie du savoir, Paris, Gallimard, 1969. 
et de contemporanéité tout en soumettant à la critique les liens causaux pouvant relier deux phénomènes historiques contigus ou synchrones.

\section{Une théorie contrefactuelle de l'imputation causale : Max Weber}

Cette présentation sommaire visait à montrer combien les questionnements sur la causalité, hérités et renouvelés, sont nombreux et variés. À chaque fois, bien que ce ne soit pas toujours explicité, il semble bien qu'une forme de raisonnement contrefactuel soit mobilisée pour penser les relations. C'est ce que suggère Max Weber, qui a exposé ce phénomène et en a tiré parti pour bâtir une véritable science historique, capable, par l'examen de «possibilités objectives », de hiérarchiser convenablement les causes. La matrice du raisonnement contrefactuel sur l'imputation causale, dont s'inspirent ensuite explicitement Raymond Aron, Paul Ricœur, Cornelius Castoriadis ${ }^{14}$, Antoine Prost ${ }^{15}$ et de nombreux «contrefactualistes» anglophones, est exposée dans son deuxième Essai sur la théorie de la science, publié en $1906^{16}$.

Weber y réfléchit sur l'imputation causale en histoire à partir des travaux d'un de ses contemporains, l'historien allemand Eduard Meyer, qui a souligné le rôle des grands hommes dans le déclenchement des guerres $^{17}$. Selon ce dernier, le déclenchement de la guerre austro-prussienne de 1866 serait la conséquence d'une décision de Bismarck. De façon commune chez les historiens, Meyer mobilise l'interrogation contrefactuelle pour la récuser immédiatement. Bismarck, écrit-il, aurait «pu prendre une autre décision, et d'autres personnalités [...] en auraient sans doute pris une autre; en conséquence, le cours de l'histoire aurait été tout autre». Mais il ajoute immédiatement dans une note de bas de page : «Il ne s'agit nullement par là d'affirmer ou de contester que dans

14. Cornelius Castoriadis, La Cité et les lois. Ce qui fait la Grèce, 2. Séminaires 1983-1984. La création humaine III, Paris, Seuil, 2008; id., Thucydide, la force et le droit. Ce qui fait la Grèce, 3. Séminaires 1984-1985. La création humaine IV, Paris, Seuil, 2011.

15. «Toute histoire est contrefactuelle. Il n'y a pas d'autre moyen, pour identifier des causalités, que de se transporter en imagination dans le passé et de se demander si, par hypothèse, le déroulement des événements aurait été le même au cas où tel ou tel facteur considéré isolément aurait été différent » écrit Antoine Prost, Douze Leçons sur l'histoire, Paris, Seuil, 1991, p. 178.

16. Max Weber, Essais sur la théorie de la science. Deuxième essai : "Études critiques pour servir à la logique des sciences de la culture» (1906), Paris, Plon, 1965.

17. Eduard Meyer, Zur Theorie und Methodik der Geschichte, Halle, Niemeyer, 1902. 
ces cas les guerres en cause n'auraient pas eu lieu; cette question est absolument insoluble et oiseuse ». Contrairement à Meyer, Weber choisit de prendre l'interrogation contrefactuelle au sérieux en se demandant ce qui aurait pu arriver si Bismarck n'avait pas pris la décision de faire la guerre. Pour Weber, cette question n'est pas vaine, elle est au contraire fondamentale en ce qu'elle concerne «le point décisif pour la structuration historique de la réalité, à savoir : quelle signification causale faut-il au fond attribuer à cette décision individuelle au sein de la totalité des éléments [causaux qui sont] infiniment nombreux ${ }^{18}$ ?» Weber poursuit sa démonstration de manière catégorique. Le salut de l'histoire réside dans l'approche contrefactuelle : «Si l'histoire prétend s'élever au-dessus d'une simple chronique des événements et des personnalités, il ne lui reste d'autre voie que celle de poser des questions de ce genre. Et pour autant qu'elle est une science, elle a toujours procédé de cette manière ${ }^{19}{ }^{\text {». }}$. Pour le sociologue allemand, c'est l'analyse contrefactuelle qui confère à l'histoire son statut de science et de toute façon les historiens ont toujours eu recours consciemment ou inconsciemment à cette démarche.

Il en fait d'ailleurs la démonstration en examinant l'analyse que fait Meyer des deux coups de feu qui provoquèrent de grands combats de rue à Berlin en mars 1848. L'historien allemand estime que la question de l'origine de ces coups de feu n'a aucune importance : "La situation était telle qu'un quelconque incident devait déclencher le conflit ${ }^{20} »$. Cette assertion implique que Meyer se soit demandé ce qui serait advenu sans ces deux coups de feu; et sa réponse est simple : la même chose. À partir de cet exemple de 1848, Weber affirme l'importance de l'approche contrefactuelle pour déterminer ce qu'il appelle la «signification historique » d'un événement.

Le jugement affirmant que, si l'on modifie ou omet en pensée un événement historique singulier dans un complexe de conditions historiques, il s'en serait suivi, en ce qui concerne certaines relations historiquement importantes, un développement différent des événements historiques, semble donc avoir une valeur considérable pour la détermination de la «signification» historique de cet événement, même si l'historien croit in praxi n'avoir à développer et à fonder consciemment et explicitement un

18. M. Weber, op. cit., p. 266.

19. Ibid., p. 267-268.

20. E. Meyer, op. cit., p. 43. 
pareil jugement que dans les cas exceptionnels, notamment lorsqu'il y a contestation à propos de cette «signification» historique. Il est évident que ce fait aurait dû susciter un examen de la nature logique de cette sorte de jugements qui portent sur les résultats auxquels il «aurait» fallu s'attendre en cas d'omission ou de modification d'un élément causal singulier dans un complexe de conditions, et inviter à une étude de leur importance pour l'histoire ${ }^{21}$.

Max Weber décide donc de développer un modèle scientifique d'analyse contrefactuelle. Ne trouvant pas d'éléments théoriques pertinents chez les historiens, qu'il dit «en détresse » sur ces questions épistémologiques, ni même chez les sociologues, il convoque les travaux du psychologue Johannes von Kries, qui est alors un des principaux théoriciens allemands des probabilités et qui a notamment forgé le concept de "possibilité objective», que va utiliser Weber ${ }^{22}$. Le sociologue élabore une méthodologie en prenant en considération les contraintes et les objectifs des historiens. Le chercheur ne peut prendre en considération l'ensemble des «causes » : c'est à la fois impossible - leur nombre est infini - et absurde, puisque l'historien cherche à expliquer exclusivement les phénomènes qui peuvent offrir un «intérêt historique». L'historien est donc contraint de sélectionner un ou plusieurs «éléments déterminants» parmi une infinité d'éléments. Subsiste un problème de taille : comment prouver l'existence d'une relation causale entre les causes sélectionnées et les effets constatés par l'historien? C'est ici qu'intervient la démarche contrefactuelle, à laquelle il donne le nom de Gedankenprozess, processus de pensée qui permet de concevoir l'imputation causale. Ce processus de pensée se fonde sur une série d'abstractions, c'est-à-dire de modifications d'un ou plusieurs «composants causatifs incontestés ${ }^{23} »$. Pour ce faire, il convient de créer des «tableaux imaginaires » (Phantasiebilder) en soustrayant un ou plusieurs faits historiques et en construisant un nouveau cours des choses. L'analyse de ces «possibilités» permet au chercheur d'identifier les causes, de les démêler et de les hiérarchiser. Ainsi, selon Weber, un événement historique est un fait «qui porte des conséquences», qui possède la capacité de provoquer un changement.

21. M. Weber, op. cit., p. 268.

22. Johannes von Kries, Über den Begriff der objektiven Möglichkeit und einige Anwendungen desselben, Leipzig, 1888.

23. M. Weber, op. cit., p. 273. 
La méthode de construction des hypothèses alternatives doit prendre en compte les «règles de l'expérience», les régularités empiriques que l'on peut identifier dans la réalité : certains éléments produisent d'une façon générale certains effets, ce que Raymond Aron dénomme «des consécutions conformes aux généralités connues». Ces calculs rétrospectifs de probabilités permettent de discerner des éléments «occasionnels», « accidentels», des tournants. Cette méthodologie contrefactuelle a été reformulée par Aron - l'un des principaux introducteurs de Weber en France - qui l'a actualisé sans doute à l'intention des historiens dans son Introduction à la philosophie de l'histoire, publiée en $1938^{24}$.

Stimulante, cette méthode contrefactuelle pose toutefois quelques problèmes. D'abord, si l'on considère que c'est le degré de probabilité qui fonde la hiérarchie des causes : comment concrètement attribuer un degré de probabilité idoine à chaque "possibilité objective» ? Il est plus facile d'«éliminer les candidats à la causalité» que d'en «couronner un seul $^{25}{ }^{2}$. D'autre part, une des principales difficultés réside dans le choix du ou des antécédents les plus pertinents, particulièrement dans le choix du moment précis qui marque le démarrage de l'enquête contrefactuelle, le fameux «turning point». Par exemple, à propos du déclenchement de la Révolution française et de la Première Guerre mondiale, il est possible d'envisager plusieurs «tournants» sans pouvoir trancher.

Si bien fondées que soient les décisions du savant, elles sont rarement les seules possibles : on aurait pu poser la question à un autre moment, car l'évolution est continue et on remonte rarement à un premier commencement; une certaine sélection des antécédents comme un certain découpage de l'effet ne s'imposent pas de manière catégorique ${ }^{26}$.

Même problème pour le niveau d'analyse : faut-il pour un même événement se situer au niveau de tel ou tel acteur, ou au contraire des phénomènes structuraux? Raymond Aron émet une autre réserve à l'endroit de la théorie de Weber. L'approche contrefactuelle suppose l'existence de «causes constantes» en histoire alors que les conditions historiques sont en perpétuelle évolution. Le risque est de s'enfermer dans

24. R. Aron, op. cit.

25. Paul Ricœur, Temps et récit, 1, L'intrigue et le récit historique (1983), Paris, Seuil, 1991, p. 329.

26. R. Aron, op. cit., p. 225. 
un schéma causal mécaniste en étudiant les «suites » d'un événement ou d'une situation plutôt que ses «transformation ${ }^{27}$ ».

Néanmoins, l'un des grands mérites de l'analyse de Weber, prolongée par ses successeurs, est de souligner l'importance logique du raisonnement contrefactuel dans la recherche des causes : on ne peut penser les relations sans imaginer des issues alternatives plus ou moins poussées, de manière plus ou moins instinctive. Différents courants de recherche ont tâché récemment de considérer ce fait en tant que tel et de tenter de modéliser ces «voies non suivies».

\section{Modélisations logiques et mathématiques du contrefactuel}

L'analyse contrefactuelle est à l'origine d'une des principales conceptions de la causalité développées au $\mathrm{xx}^{\mathrm{e}}$ siècle. Aux États-Unis elle a été formulée par le philosophe David Lewis, dans son ouvrage au titre explicite, Counterfactuals, paru en $1973^{28}$. Lewis réduit la relation de causalité entre deux phénomènes à l'existence d'une chaîne causale constituée d'une série finie d'événements. On peut dire que l'événement $\mathrm{C}$ dépend causalement de l'événement A si les deux contrefactuels suivants sont vrais : si A avait eu lieu, C aurait eu lieu; si A n'avait pas eu lieu, $\mathrm{C}$ n'aurait pas eu lieu ${ }^{29}$. Philosophe analytique, David Lewis a poursuivi jusqu'au bout les implications de la logique modale : les contrefactuels permettent de réfléchir à des événements non réalisés dans notre monde mais qui, sous d'autres conditions, auraient pu advenir ailleurs. La définition de ces mondes possibles, causalement séparés les uns des autres, autorise le chercheur à effectuer des comparaisons et à tirer des conclusions distinguant le vrai du faux. Dans cette perspective, ces autres mondes ne sont pas des constructions de l'imaginaire, mais constituent d'autres réalités concrètes ${ }^{30}$.

L'ouvrage de Lewis a fait grand bruit, suscitant un certain nombre de critiques, mais également de l'intérêt, chez les philosophes ou les littéraires. Spécialiste de littérature comparée, Thomas Pavel l'utilise par exemple

27. Ibid., p. 226.

28. David Lewis, Counterfactuals, Oxford, Blackwell, 1973.

29. Id., «Causation», The Journal of Philosophy, 70/17 (1973), p. 556-567.

30. Id., On the plurality of worlds, Oxford, Blackwell, 1986. 
pour discuter les différences entre mondes fictionnels et mondes réels ${ }^{31}$. Pour les philosophes Frédéric Nef et Pierre Livet, l'analyse contrefactuelle permet de saisir une dimension essentielle de la vie sociale ${ }^{32}:$ les activités sociales dépendent en partie de contrefactuels : «de faits qui ne sont plus, de faits qui ne sont pas encore, d'états de choses dont l'existence est évitée ou qu'on n'a pas réussi à faire passer dans l'existence ${ }^{33}$ ». Son usage est plus délicat pour les sciences sociales, méfiantes face à l'idée d'une réalité structurée par des logiques modales auto-centrées. Le «réel», objet de toutes les interrogations, qu'il soit partagé, fantasmé, construit, naturalisé, subi, reste un horizon d'attente à découvrir plutôt qu'un espace apparaissant comme préconstruit ${ }^{34}$.

Une autre forme de modélisation est mathématique, qui entend établir des relations démontrables entre des éléments strictement définis. Il n'est donc pas étonnant que les travaux qui mobilisent le plus les outils mathématiques ou statistiques posent explicitement la question contrefactuelle, dans la mesure où elle est de toutes façons produite par la démarche. Celle-ci peut-être écartée bien entendu, mais elle peut également être utilisée comme élément d'une démonstration.

Il est inutile de revenir sur les savantes projections cliométriques ou démographiques, évoquées ailleurs ${ }^{35}$. Cette approche mathématique a inspiré le programme de recherches de Gary King et Langche Zeng sur les processus de démocratisation dans le monde, les « effets causaux » de la démocratie et les conséquences des opérations onusiennes de consolidation de la paix. Ces chercheurs ont constitué aux États-Unis des bases de données sur l'ouverture des marchés, les taux de mortalité infantile, le degré de démocratie (autocratie, démocratie partielle, démocratie complète), le pourcentage de la population active dans le domaine militaire

31. Thomas Pavel, Fictional Worlds, Cambridge, Harvard University Press, 1986.

32. Pierre Livet et Frédéric Nef, Les êtres sociaux. Processus et virtualité, Paris, Hermann, 2009.

33. P. Livet, «La substituabilité comme propriété des êtres sociaux, les conditionnels et la prédication», Igitur-Arguments philosophiques, $2 / 2$ (2011), p. 1-13. Voir aussi sa contribution au présent numéro. 34. Saul Kripke a élaboré une théorie de la sémantique des mondes possibles qui apparaît plus ouverte dans son approche, et possède des ressources intéressantes : Naming and Necessity, Cambridge, Harvard University Press, 1980 (traduction par Pierre Jacob et François Recanati, La logique des noms propres, Paris, Minuit, 1982).

35. Voir l'article d'Isabelle Drouet, Stéphanie Dupouy, Laurent Jeanpierre et Florian Nicodème dans ce numéro. 
et l'efficacité législative ${ }^{36}$. Cette quête de contrefactuels plausibles passe par des calculs très sophistiqués : King et Zeng ont récemment proposé la formule $\mathrm{E}(Y / X)=g(X$, beta) afin de distinguer les contrefactuels «produits par le modèle» (indépendamment des données) de ceux qui correspondent aux données empiriques disponibles ${ }^{37}$. Cette formule permet d'affiner les précédentes analyses et de démontrer scientifiquement, comme ils le pensaient, la faiblesse des probabilités que l'Arabie saoudite devienne une démocratie et le Canada une autocratie ${ }^{38}$. Plus sérieusement, ils ont pu déconstruire les résultats d'autres travaux de sciences politiques, notamment ceux consacrés aux effets des opérations de consolidation de la paix menées par les Nations unies ${ }^{39}$. D'après la formule de King et Zeng, tous les résultats produits sont «dépendants du modèle», donc inutiles.

En dépit de ces raffinements, on mesure rapidement les limites de ce type d'approche, qui renvoient à des débats méthodologiques classiques. D'abord, dans le cadre d'analyse de ce logiciel WhatIf, le nombre de cas indépendants du modèle se révèle très faible. Et, plus largement, le modèle traduit une fois encore une conception très discutable du devenir historique : les catégories mobilisées pour classer les pays sont insuffisamment définies, les relations établies entre elles ne sont pas nécessairement causales, et, bien que calculé par «années de pays», le temps reste extérieur au modèle puisque ce dernier ne se modifie pas. De fait, ces outils empêchent de penser ce qu'est une démocratie comme processus historique, et éludent un ensemble de facteurs locaux, sociaux et culturels au rôle tout aussi important. Le test contrefactuel nourrit ici une conception très positiviste, au sens restreint du terme, du monde social.

On l'a dit, toute modélisation au sens large, lorsqu'elle définit statistiquement ou empiriquement un «chemin» à suivre, repose par définition sur un raisonnement de type contrefactuel : le chercheur évalue, même

36. Gary King et Langche Zeng, «Improving Forecasts of State Failure », World Politics 53/4 (2002), p. 623-658. URL : http://gking.harvard.edu/files/abs/civil-abs.shtml (dernière date d'accès aux pages Web : 10 septembre 2012).

37. Idem, «When Can History Be Our Guide? The Pitfalls of Counterfactual Inference», International Studies Quaterly, 51 (2007), p. 183-210. URL : http://gking.harvard.edu/files/abs/counterf-abs.shtml 38. Cela signifie que le monde n'a pas connu de situation antérieure correspondant à ce cas de figure, et donc que «l'histoire peut être notre guide». C'est le principe de la démarche envisagée : comparer les issues possibles aux diverses situations avérées du passé.

39. Michael W. Doyle et Nicholas Sambanis, «International Peacebuilding: A Theoretical and Quantitative Analysis», American Political Science Review, 94/4 (2000), p. 779-801. 
inconsciemment, ce qui aurait dû se passer si toutes les conditions avaient été remplies. Ainsi en histoire économique et sociale, toutes les analyses centrées sur la «Path Dependency» ou sur la «révolution industrielle», qui par contrecoup définissaient des «voies singulières» françaises et autre «Sonderweg» allemand, étaient bien des types de raisonnement contrefactuel. Ce faisant, ils autorisaient les comparaisons mais masquaient aussi certains aspects de la complexité des situations comme des enchaînements. D'ailleurs, la tension entre ces deux possibilités n'est pas résolue aujourd'hui.

\section{Anti-déterminisme et explicitation}

Un des moyens de lutter contre ces catégories englobantes, lorsqu'elles structurent trop fortement la perception des sociétés, est paradoxalement de recourir aussi à un raisonnement contrefactuel, afin cette fois de «casser» les enchaînements causaux implicites de ces schémas. C'est ce qu'a proposé Kenneth Pomeranz dans sa célèbre analyse comparée des mutations économiques européennes et chinoises ${ }^{40}$. De fait, le raisonnement contrefactuel peut être mobilisé pour critiquer la force des liens établis a priori et cette fois remettre en cause les schémas historiques déterministes.

L'un des premiers à saisir ce potentiel subversif de l'approche contrefactuelle est l'historien Paul Lacombe dans son ouvrage De l'Histoire considérée comme science, paru en 1894. Lacombe dénomme cette démarche «l'expérience imaginaire» :

Supposer par la pensée à une série d'événements une tournure autre que celle qu'ils eurent, refaire par exemple la Révolution française. Beaucoup d'esprits trouveront sans doute que cela constitue un ouvrage vain, sinon même dangereux. Je ne partage pas ce sentiment. Je vois un danger plus réel dans la tendance qui nous porte à croire que

40. Kenneth Pomeranz, The Great Divergence. China, Europe, and the Making of the Modern World Economy, Princeton, Princeton University Press, 2000. La dimension proprement contrefactuelle est précisée dans : id., «Without Coal? Colonies? Calculus? Counterfactuals and industrialization in Europe and China», dans Philip Tetlock, Richard Lebow, Geoffrey Parker (dir.), Unmaking the West, "What-If?" Scenarios that Rewrite World History, Ann Arbor, University of Michigan Press, 2006, p. 241-276. 
les événements historiques ne pouvaient pas être autrement qu'ils n'ont été. Il faut se donner au contraire le sentiment de leur instabilité vraie. Imaginer l'histoire autrement qu'elle ne fut sert d'abord à cette fin ${ }^{41}$.

Raymond Aron ne dit pas autre chose dans son Introduction à la philosophie de l'histoire. La première et la plus importante des fonctions de l'histoire contrefactuelle est de lutter contre le déterminisme : «l'enquête causale de l'historien a moins pour sens de dessiner les grands traits de relief historique que de conserver ou de restituer au passé l'incertitude de l'avenir ${ }^{42}{ }$. Il ajoute plus tard dans son ouvrage sur les Dimensions de la conscience historique : «Les constructions irréelles doivent rester partie intégrante de la science, même si elles ne dépassent pas une vraisemblance équivoque, car elles offrent le seul moyen d'échapper à l'illusion rétrospective de la fatalité ${ }^{43}{ }^{\prime}$. Les historiens construisent des causes qui a posteriori confèrent une nécessité au déroulement des événements en contredisant «l'impression contemporaine de contingence ${ }^{44}$ ».

Dans la même lignée, explicitement inspirée d'Aron, le philosophe Paul Ricœur a insisté sur la nécessité de reconstituer l'horizon d'attente du passé en mettant au jour les possibilités probables, afin de restituer l'incertitude de l'événement :

Il faut comprendre que l'opération imaginaire par laquelle l'historien suppose par la pensée un des antécédents disparus ou modifiés, puis tâche de construire ce qui se serait passé dans cette hypothèse, a une signification qui dépasse l'épistémologie. L'historien se comporte ici en narrateur qui redéfinit par rapport à un présent fictif les trois dimensions du temps. Rêvant d'un événement autre, il oppose l'uchronie à la fascination du révolu. L'estimation rétrospective des possibilités revêt ainsi une signification morale et politique, qui excède sa signification purement épistémologique : elle rappelle aux lecteurs d'histoire que "le passé de l'historien a été le futur des personnages historiques". Par son caractère probabiliste, l'explication causale incorpore au passé l'impré-

41. Paul Lacombe, De l'Histoire considérée comme science, Paris, Hachette, 1894, p. 63-64. 42. R. Aron, op. cit., p. 224.

43. Id., Dimensions de la conscience historique, Paris, Plon, 1961, p. 186-187.

44. Id., Introduction à la philosophie..., op. cit., p. 224. 
visibilité qui est la marque du futur et introduit dans la rétrospection l'incertitude de l'événement ${ }^{45}$.

L'usage du contrefactuel relève encore ici de la recherche causale, mais il sert à ouvrir le champ des causalités plutôt qu'à déterminer et à hiérarchiser les causes.

Si les philosophes de l'histoire ont tenté d'appréhender la question de l'imputation causale, cette dernière pose problème aux historiens. L'antiquisant Paul Veyne est un des rares à s'être attelé à ce problème dans Comment on écrit l'histoire. Il part d'un constat fort simple : l'historien n'ayant accès directement qu'à une part infime des informations nécessaires pour faire comprendre le passé, «pour tout le reste, il lui faut boucher les trous $^{46}{ }$. Il dénomme cette opération de remplissage la «rétrodiction» et l'explique par une hypothèse historique simple : «Louis XIV devint impopulaire parce que les impôts étaient trop lourds ». Cette assertion peut résulter de deux raisonnements très différents. En effet, soit l'historien sait par les sources que la fiscalité a bien été la cause de l'impopularité du souverain, soit il dispose de deux informations distinctes : il sait que les impôts étaient lourds et que, par ailleurs, Louis XIV est progressivement devenu impopulaire. Dans ce cas, le chercheur effectue une «rétrodiction », c'est-à-dire qu'il remonte de l'impopularité constatée à une cause présumée, l'excessive pression fiscale. Le problème du discours historique réside dans le passage constant et souvent inconscient d'un raisonnement à l'autre, sans que le statut de la preuve soit explicité.

Bien que Paul Veyne, comme Max Weber, emprunte cette notion de «rétrodiction » à la théorie des probabilités, il souligne le caractère irrégulier, partiel et restreint de l'imputation causale en histoire, surnommée la "causalité sublunaire», parce que chaque situation historique est spécifique et que l'historien ne peut jamais prendre en considération l'ensemble des causes. Dans son étude sur la «rétrodiction», Veyne pressent bien que la méthode contrefactuelle qu'il appelle «prédiction historique» peut aider l'historien à accroître sa compréhension de la situation historique :

Exemple de prédiction historique : demandons-nous ce qui serait arrivé si Spartacus avait battu les légions romaines et était devenu le maître

45. P. Ricœur, op. cit., p. 331-332.

46. Paul Veyne, Comment on écrit l'histoire. Essai d'épistémologie, Paris, Seuil, 1974, p. 194. 
de l'Italie du Sud; la fin de l'esclavagisme? Le passage à un cran supérieur dans l'ascension des rapports de production? Un parallèle suggère une meilleure réponse, que tout ce que nous savons du climat de l'époque semble confirmer; depuis que nous avons appris qu'une génération avant Spartacus, lors de la grande révolte des esclaves de Sicile, ces révoltés s'étaient donné une capitale et un roi, nous pouvons estimer que, si Spartacus avait gagné, il aurait fondé en Italie un royaume hellénistique de plus, où, assurément, l'esclavage aurait existé, comme il existait partout à cette époque ${ }^{47}$.

Paul Veyne ne poursuit pas plus avant sa réflexion contrefactuelle et préfère établir les conditions d'une «rétrodiction» pertinente. Le propos devient flou lorsque l'auteur distingue les domaines où l'on peut effectuer une «rétrodiction» parce qu'il existe quelques constantes sur lesquelles l'historien peut se fonder (coutumes, codes linguistiques, violence, etc.), et des domaines d'étude moins propices (histoire de l'art et des idées). Ces partages sont discutables et la recherche causale difficile. L'auteur a par la suite choisi de privilégier l'«explicitation» des situations plutôt que l'«explication ${ }^{48} »$. Ce faisant, s'il le fait convenablement, il peut faire saillir l'abondance des relations causales, a priori insaisissables. En dégageant ces liens, en s'attachant autant à la singularité des contenus qu'à leurs enchevêtrements, l'historien aide à pénétrer la complexité des époques passées et invite au décentrement comme à la remise en cause de ses catégories d'analyse. La position, suivie par de nombreux historiens, souvent inspirés par l'anthropologie culturelle de Clifford Geertz, est très stimulante. Elle a le mérite de contourner le problème ardu de la causalité tout en encourageant la connaissance des sociétés passées par une tentative d'imprégnation nourrie des sources, par l'analyse du point de vue des acteurs et par l'interprétation du chercheur.

\section{Configurations, bifurcations et réalités non linéaires}

Récemment, d'autres travaux ont proposé des approches plus composites des enchaînements causaux ou de l'explication historique, en tentant

47. Ibid., p. 202-203.

48. P. Veyne, Le Quotidien et l'intéressant, Paris, Les Belles Lettres, 1994. 
d'articuler à nouveaux frais régularités et changements, stabilité et irruptions, relations et hétérogénéité.

Il peut paraître étonnant de convoquer ici les travaux de Norbert Elias : son modèle processuel est ancien, et peine précisément à penser les événements, ruptures et autres «tournants». Le sociologue s'est en outre fermement opposé à l'explication causale, dès 1939, en réponse à une remarque de Raymond Aron. La recherche causale traditionnelle, un des modes de raisonnement dominants, suppose en effet selon lui des jugements de valeur implicites :

On croit ainsi "expliquer" le chant d'amour courtois en disant qu'il provient de la poésie lyrique mariale (ou éventuellement aussi du contact avec la culture arabe). Mais l'on ne s'interroge pas sur les raisons du mouvement par lequel la poésie lyrique mariale (ou ce que cela peut bien être) se transforme en poésie lyrique courtoise. Et ainsi risque-ton peut-être de mal comprendre ma démonstration selon laquelle certains aspects du code de conduite civilisée occidental se sont d'abord développés dans les cercles de la noblesse de cour et ont ensuite été repris, assimilés et retravaillés par les cercles bourgeois - en particulier en France -; on risque en effet de comprendre que j'estime que la noblesse de cour est la cause ou l'initiatrice de la vague de civilisation bourgeoise qui a succédé. [...] Le processus que nous avons sous les yeux ressemble $[\ldots]$ à certains processus chimiques où, dans un liquide en voie de restructuration moléculaire, par exemple de cristallisation, la forme cristalline affecte d'abord un petit noyau central autour duquel s'opère la fixation cristalline. Rien ne serait plus faux que de considérer le noyau comme la cause de la cristallisation ${ }^{49}$.

Cela ne signifie pas pour autant qu'il récuse tout projet d'explication. Les analyses causales, telle qu'il les connaît à cette période, lui paraissent trop unidimensionnelles. Il préfère mettre en avant les relations entre les éléments d'une société, ainsi que leurs dynamiques. C'est ce qu'il appelle une approche «configurationnelle», puis «figurationnelle», pour souligner combien l'association entre les éléments n'est pas nécessairement homogène : le sociologue doit saisir un état de ces interrelations. Son

49. «Un échange de lettres entre Raymond Aron et Norbert Elias (juillet 1939)» (présenté et traduit par Marc Joly), Vingtième Siècle. Revue d'histoire, 106/2 (2010), p. 97-102. 
«processus de civilisation» correspond parfaitement à cette perspective. Il désigne un ensemble de dynamiques qui se nourrissent l'une l'autre : le développement de l'État dans sa forme dite «moderne » (monopolisation de la violence, impôt), l'accroissement des interdépendances liées à la différenciation des activités et l'émergence d'une nouvelle économie psychique caractérisée par un meilleur autocontrôle. Tous ces processus vont ensemble sans que l'un soit la «source» de l'autre. La démarche, comprise ainsi, n'est d'ailleurs pas insensible à l'événement. Dans cette perspective, un «événement» (et c'est ce qui le définit comme tel) va modifier la relation antérieure entre les éléments et les dynamiques pour proposer un nouvel agencement, une nouvelle «configuration».

Ce faisant, les configurations éliassiennes ne sont pas fermées à la question des possibles du passé, dans la mesure où elles dessinent des rapports de force dynamiques dont l'issue, sans être hasardeuse, n'est pas donnée. Il s'agit, répète-t-il souvent, de «processus non planifiés», qui obéissent à une direction qui peut être changeante. L'un de ses leitmotivs, lorsqu'il entend faire se rejoindre rigueur scientifique et intérêt politique de son analyse, peut d'ailleurs se résumer ainsi : si les hommes avaient mieux connu ou connaissaient mieux (grâce à sa sociologie processuelle) la situation d'interdépendance dans laquelle ils sont pris, alors ils auraient fait ou feraient de meilleurs choix. Sans cela, ils risquent de s'enfermer dans de mauvaises décisions menaçant à terme le processus de civilisation ${ }^{50}$.

Dans les années 1980, Elias s'est d'ailleurs intéressé à la question de l'utopie. Dans l'un de ses articles, non traduit en français, il explique que, lorsque Thomas More développe son «utopie», il s'agit d'une réponse mentale à une situation sociale, politique mais également technologique donnée, qui correspond à un moment particulier du processus de civilisation : celui où l'espoir ou la crainte ne se manifestent plus seulement dans des figures comme l'enfer ou le paradis, mais nourrissent une disposition mentale où l'on commence à penser possible de changer le monde ${ }^{51}$. Au $\mathrm{xx}^{\mathrm{e}}$ siècle, constate-t-il, les utopies proposées (Huxley, Orwell) ne sont plus positives, mais négatives : elles expriment une crainte et une menace. Or le fait est paradoxal dans la mesure où les hommes ont désormais

50. Norbert Elias, Engagement et distanciation. Contributions à la sociologie de la connaissance, Paris, Fayard, 1993.

51. Id., «Thomas Morus' Staatkritiks. Mit Überlegungen zur Bestimmung des Begriffs Utopie», dans Wilhelm Voßkamp (dir.), Utopieforschung. Interdisziplinäre Studien zur neuzeitlichen Utopie, vol. 2, Stuttgart, Metzler, 1982, p. 101-151. 
les moyens au moins techniques de résoudre la plupart des problèmes auxquels ils font face : ils peuvent par exemple réaliser ce vieux rêve qui est d'aller sur la Lune ou résoudre la question de la faim dans le monde. Cette situation nouvelle, ces possibilités neuves, mal saisies, font naître un sens de la responsabilité qui entraînerait une angoisse particulière et un sentiment d'insécurité : les hommes ne savent pas quoi faire de leurs capacités inédites de réalisation des utopies. Comme toujours, Elias historicise son sujet et l'inscrit à la fois dans la durée et dans les contextes sociaux, culturels et politiques mouvants. Ainsi l'ampleur des possibles, qui varie dans le temps, est aujourd'hui maximale : sans doute se dessine-t-il là un autre élément à prendre en compte pour comprendre le développement actuel des pensées contrefactuelles ${ }^{52}$. De fait, les configurations éliassiennes, inscrites dans la très longue durée, permettent d'appréhender simultanément des ensembles de possibles historiquement contraints et mouvants. En ce sens, il propose en quelque sorte des contrefactuels encodés dans le processus social, fruit des interdépendances spécifiques à une société donnée. Si ses modalités d'application restent floues, cette manière de faire qui s'applique aussi bien à l'échelle individuelle qu'à celle d'une époque semble stimulante.

D'autres perspectives rompant avec les modalités plus classiques d'enchaînement des causes ont été développées. C'est le cas des analyses sociologiques en termes de «bifurcations», qui invitent à combiner les régularités à l'œuvre dans certains espaces sociaux avec la possibilité des basculements, selon une logique hétérogène ${ }^{53}$.

L'étude des parcours de vie, développée par la sociologie interactionniste américaine à partir des années 1950, est à l'origine de cette perspective. Ces travaux considèrent qu'au cours d'une vie, un même individu est pris dans des univers sociaux cohérents, avec leurs normes et leurs dispositions qu'il faut intégrer. Chaque individu peut basculer de l'un vers l'autre (de l'école à l'université, de l'université au monde du

52. Un aspect que l'on retrouve également, selon lui, à l'échelle individuelle : «Il est dans la nature même des sociétés qui exigent de l'individu un plus ou moins haut degré de spécialisation de lui faire négliger une foule de possibilités qu'il n'utilise pas, de vies qu'il ne vit pas, de rôles qu'il n'aura pas joués, d'expériences qu'il n'aura pas vécues et d'occasions qu'il aura manquées » (La Société des individus, Fayard, 1991, p. 180).

53. Marc Bessin, Claire Bidart et Michel Grossetti (dir.), Bifurcations. Les sciences sociales face aux ruptures et à l'événement, Paris, La Découverte, 2010. 
travail, d'un milieu social à un autre ${ }^{54}$...). Cela suppose alors une adaptation qui engage autant les actions que l'attitude, voire les manières de penser. Les études de ce type sont nombreuses, qu'elles concernent le monde des élites ou celui des délinquants. Pour ces derniers, des travaux ont ainsi montré l'importance, dans des parcours compliqués, faits de petits boulots et de petite délinquance, de certains événements (mariage, travail stable), véritables tournants permettant de modifier durablement la situation et les comportements adoptés ${ }^{55}$. Si un tel événement a lieu, alors la personne bascule dans un cadre de vie et un type d'actes entièrement différents. Dans ces cas, le nombre de voies - notamment ascendantes est certes limité, notamment par l'organisation du marché du travail; mais la pluralité des voies et des passages existe bien. Une telle approche est bien entendu délicate à mettre en œuvre en histoire dans la mesure où il faudrait avoir en main l'ensemble des informations permettant de dessiner convenablement ces espaces sociaux.

Les analyses concernant les événements sont assez proches. Ces derniers sont considérés de plus en plus comme un temps d'ouverture des possibles, dans lequel les pesanteurs antérieures demeurent, même si elles agissent différemment. Ces travaux peuvent évoquer les perspectives de l'histoire contrefactuelle de Niall Ferguson ${ }^{56}$, mais pour la critiquer : cette méthode proposerait des scenarii qui typifient excessivement les autres issues possibles et manqueraient ainsi l'essentiel, c'est-à-dire la plasticité des structures propre à l'«événement ${ }^{57}$ ». Cela dit, le raisonnement mis en œuvre pour penser ces moments est bien de type contrefactuel - et cette subtilité est d'importance ici. Écoutons William Sewell Jr, discutant l'analyse par Mark Traugott du massacre de juin 1848. Selon ce dernier, le fait qu'une partie des ouvriers était d'un côté de la barricade, avec les insurgés, et une autre, avec les gardes mobiles, prête à les massacrer

\footnotetext{
54. Everett C. Hugues, «Cycles, Turning Points and Careers», dans The Sociological Eye, Chicago, Aldine, 1971, p. 124-131.

55. Robert J. Sampson et John H. Laub, Crime in the Making : Pathways and Turning Points Through Life, Cambridge, Harvard University Press, 1993.

56. Niall Ferguson est l'un des historiens anglophones qui ont redonné une visibilité à la démarche contrefactuelle dans les années 1990, en la mobilisant contre l'historiographie d'inspiration marxiste : N. Ferguson (dir.), Virtual History. Alternatives and Counterfactuals, Londres, Papermac, 1997. 57. Voir les mises en garde de Michel Dobry, «Le politique dans ses états critiques : retour sur quelques aspects de l'hypothèse de continuité», dans M. Bessin, C. Bidart et M. Grossetti (dir.), op. cit., p. 64-88; sur l'événement comme ouverture des possibles, Alban Bensa et Éric Fassin, «Les sciences sociales face à l'événement». Terrain, 38 (2002), p. 5-20.
} 
s'explique non par le type de métier exercé auparavant mais par les organisations dans lesquelles ces personnes ont été prises entre février et juin 1848 (Ateliers nationaux et garde mobile). «Si le gouvernement avait maintenu les Ateliers [nationaux] en activité et conservé Thomas à leur direction, peut-être que l'insurrection ne se serait jamais produite». Mais «l'adoption d'une temporalité événementielle par Traugott ne signifie pas qu'il a abandonné la sociologie pour l'histoire narrative ${ }^{58} \gg$. De fait, poursuit-il, Traugott propose une analyse à la fois «path dependent», causalement hétérogène, et contingente, dans laquelle l'action sociale joue un rôle décisif dans la reconfiguration des structures. Pour Sewell, l'essentiel pour la sociologie événementielle qu'il promeut est donc d'identifier des possibles contraints et des causalités non linéaires. Et, dans cette perspective, le raisonnement contrefactuel, quoique non explicité en tant que tel, est encore un élément à part entière de l'analyse, conjugué avec l'analyse des structures dans une perspective dynamique.

Le sociologue Andrew Abbott est probablement celui qui a poussé le plus loin ce type d'approche de la causalité en sciences sociales. Spécialiste des professions, puis des disciplines académiques, nourri autant de l'interactionnisme goffmanien que de la longue durée braudélienne, Andrew Abbott a en outre longtemps mobilisé les outils statistiques les plus sophistiqués avant de les soumettre à la critique. On y retrouve les griefs énoncés plus haut : la catégorisation est discutable, la relation toujours identique entre les éléments, le temps et le contexte sont absents : tout en soulignant l'apport réel des statistiques, il appelle la réalité qu'elles produisent la «réalité linéaire générale ${ }^{59}$ ». Abbott entend résoudre la tension entre approche quantitative et approche narrative, entre causalité et temporalité, entre formalisation et interprétation. Le «présent» peut être défini comme un moment où rien ne change, avec sa structure et ses régularités observables par le sociologue. Ce moment s'inscrit toutefois dans des changements plus larges, dont on peut observer à une autre échelle la structure et les régularités, qui s'insèrent sans doute elles aussi dans des mutations plus lentes, etc. Tout cela définit des configurations de présents associés entre eux, offrant des possibilités et des impossibilités

58. William H. Sewell (Jr.), «Trois temporalités : vers une sociologie événementielle» (traduit par M. Grossetti), dans M. Bessin, C. Bidart et M. Grossetti (dir.), op. cit., p. 109-146, ici p. 136.

59. Andrew Abbott, «Transcending General Linear Reality», dans Time Matters. On Theory and Method, Chicago, University of Chicago Press, 2001. 
évoluant dans le temps. Pour éclairer cela, Abbott analyse l'exemple de l'invention du harpon à grenade par le Norvégien Svend Foyn, entre 1860 et $1880^{60}$. Bien des modèles de harpon de ce type avaient été élaborés auparavant, dès les années 1820. Néanmoins, celui de Foyn s'impose car il s'inscrit dans une période de basculement structurel de la pêche à la baleine : les bateaux se mécanisent davantage, la pression du marché est plus grande, etc. Proposée plus tôt, l'«invention» eût échoué. Le sociologue cherche en fait à combiner les possibilités offertes par les mutations structurelles larges et la manière dont elles vont se réaliser ou non, au niveau de la vie de l'individu.

Significativement, Andrew Abbott fait du raisonnement contrefactuel un des «trucs» qui permettent de mieux penser les problèmes sociaux ${ }^{61}$. Mais il s'agit, tel qu'il a été exploité par Niall Ferguson, d'un «truc» un peu grossier. Toutefois ce qui peut être formulé comme la question des voies non advenues, des «possibilités futures ${ }^{62}$ », est essentiel dans sa conception des causalités réelles, discontinues et historicisées. Cette perspective est précisée dans un texte consacré aux turning points où il évoque la diversité des points de bifurcations qui constituent en réalité des processus plus ou moins longs et peuvent engendrer des ruptures brutales comme des transitions lentes d'un régime de probabilité à un autre ${ }^{63}$. Mais l'essentiel est ailleurs. Il faut en effet considérer que «le changement est l'état normal des choses», et que les permanences sont des constructions à interroger. À partir de là, le sociologue développe une «pensée par configurations ${ }^{64}{ }^{\star}$ qui prend en considération des «agencements de singularités complexes». Lorsqu'ils sont marqués par de relatives régularités, ces derniers s'apparentent à ce que l'on appelle couramment des «structures». Ce faisant, ces structures, tout en étant un lourd facteur d'inertie sont faites de tensions. Elles évoluent et dessinent ainsi des possibilités structurelles de changements, qui peuvent ou non être mobilisées par les individus ou les groupes selon les circonstances. Pour saisir l'idée, Abbott compare l'action de ces individus ou groupes sociaux à une clé, la situation à une serrure. Dans cette perspective, le changement fait partie de la structure sociale et le turning point est un

60. Id., «Temporality and Process in Social Life», dans Time Matters..., op. cit., p. 219-239.

61. Id., Method of Discovery : Heuristics for the Social Sciences, New York, Norton, 2004.

62. Ibid., p. 234.

63. A. Abbott, «On the Concept of Turning Point», Comparative Social Research, 16 (1997), p. 85-105.

64. On peut noter la proximité avec la position de Norbert Elias évoquée plus haut. 
moment court entraînant la réorientation d'un processus. Les autres possibles, en nombre limité, sont inclus dans la structure elle-même.

L'intérêt de l'approche d'Andrew Abbott est par ailleurs de ne pas avoir abandonné son exigence modélisatrice. Il a ainsi élaboré un outil statistique, appelé « appariement optimal» (optimal matching), qui permet de réunir les données selon cette orientation. Le logiciel, selon un système de glissements entre les trajectoires et d'évaluation des fréquences, permet de proposer des types de séquences probables et improbables. Il permet ainsi, dans une réalité sociale multiple et incertaine, de déterminer des séquences cohérentes et des relations mouvantes entre elles ${ }^{65}$. Les usages, variés, peuvent ainsi servir à dessiner un espace des possibles et des contraintes qui apparaît comme une définition élargie des situations. L'outil a ainsi été mobilisé récemment par différents chercheurs comme Nicolas Mariot et Claire Zalc qui, à partir d'une base de 991 juifs de Lens, ont pu visualiser l'espace mouvant des possibilités d'échapper ou non à la déportation ${ }^{66}$. Entre 1940 et 1942 , les possibilités sont importantes, les tentatives de les exploiter faibles; à partir de 1944, la volonté de fuite est plus grande, mais les possibilités très réduites. L'outil, en corrélation avec le projet précédent, permet ainsi de déterminer les champs d'autres issues possibles et de voir comment ils se modifient, qu'ils soient utilisés ou non. Comme le précisent d'ailleurs Abbott et ses épigones, l'optimal matching ne donne en aucun cas une vision exacte de la «réalité». Il constitue juste un moyen de mieux apprécier un problème et les informations complexes recueillies. Cette approche peut parfaitement être menée de manière plus qualitative lorsque les informations disponibles sont lacunaires.

Cette approche peut susciter des critiques pertinentes. Chez Abbott par exemple, la question du langage, de la mise en mots des faits et de leurs significations a posteriori, n'intervient pas (ou peu); de même est presque absente la question de l'intériorisation par les acteurs ou les groupes sociaux des expériences antérieures et des futurs non advenus modifiant la perception des possibles et donc leur mobilisation. Mais le plus important est qu'Andrew Abbott a cherché à intégrer de manière

65. Claire Lemercier, «Les carrières des membres des institutions consulaires au XIX ${ }^{\mathrm{e}}$ siècle», Histoire et mesure, 20/1-2 (2005), p. 59-95.

66. Nicolas Mariot et Claire Zalc, Face à la persécution. 991 Juifs dans la guerre, Paris, Odile Jacob et Fondation pour la mémoire de la Shoah, 2010. 
scientifique le non-advenu (et donc le changement) dans la structure sociale elle-même : c'est ce qu'il appelle le «positivisme narratif». La causalité, chez lui, est donc à la fois régulière et irrégulière, micro et macro, historique et sociologique. Dans un tel cadre, la démarche contrefactuelle, plus contrôlée, peut cette fois participer à la détermination de causalités socialement hétérogènes, historiquement variables, inscrites dans des processus de déterminations pluriels. Dès lors, elle ne semble plus seulement un outil extérieur, mais un élément à part entière de la perception du monde social que les chercheurs tâchent d'objectiver.

Ainsi s'impose la diversité des usages possibles du contrefactuel dans la recherche des causalités, qu'elles soient déterministes ou contingentes, linéaires ou multiples, inscrites sur un même plan temporel ou à l'articulation d'échelles discontinues de détermination. Cela ne doit pas étonner : la démarche contrefactuelle est par définition une pensée non prédéfinie des relations. Elle permet bien aux chercheurs de réfléchir à nouveau sur la causalité, le déterminisme et la contingence en histoire.

Le récit historique est ainsi souvent structuré par la succession d'événements fondateurs, de «turning points » qui déterminent la suite du devenir historique. Toutefois, tout comme il n'existe pas d'événement contingent en soi, il n'y a de tournant que dans le cadre d'un système d'explication particulier. Les «turning points» sont le produit du paradigme historique, du modèle narratif et de la vision du monde du chercheur ${ }^{67}$. Ces tournants historiques ainsi que le phénomène de la contingence appartiennent à des procès narratifs au service d'une philosophie de l'histoire ${ }^{68}$. Les historiens ont besoin de sélectionner et d'articuler chronologiquement des événements et des structures pour bâtir une intrigue convaincante. Bien souvent, le chercheur élude l'analyse des «causes» au profit de la description des «effets» et, dans ce cas, l'analyse contrefactuelle nous permet de débusquer les modèles théoriques sous-jacents qui informent le discours historique. En adoptant cette méthode, l'historien peut démontrer que son récit est plus plausible que toutes les autres histoires contrefactuelles et alternatives envisagées. Ainsi l'opération contrefactuelle

67. Wolfgang Knöbl, Civilizational Analysis and the Problem of Contingency, Working Papers, Centre on Migration, Citizenship and Development, 82, 2010.

68. Hayden White, Metahistory. The Historical Imagination in Nineteenth-Century Europe, Baltimore, The Johns Hopkins University Press, 1973, p. XI. 
est-elle nécessaire pour penser le changement et la permanence. Elle ne garantit en rien la rigueur ou la justesse des relations ainsi déterminées; en revanche, elle peut aider à appréhender des relations enchevêtrées et à sortir des schèmes causalistes contraignants sans renoncer à la question des déterminations.

Au cours de cet examen, le questionnaire est par ailleurs imperceptiblement passé de la quête des causalités au problème des futurs possibles du passé. De même, la dimension «scientifique» de l'analyse contrefactuelle s'est imprégnée peu à peu de problématiques ayant davantage trait à l'intrigue et à l'usage de l'imagination en histoire ${ }^{69}$. Ce glissement se conçoit aisément : les deux paradigmes de la science sociale - l'explicatif et l'interprétatif, le modèle et le récit - se croisent et se mêlent sans $\operatorname{cesse}^{70}$. De plus, dans le cadre de la recherche historique, fondée sur la rencontre avec l'«estrangement» porté par l'archive, le recours à cette démarche ne peut être trop figé ou catégorique. Cela explique peut-être également les difficultés qu'il y a à suivre un raisonnement qui paraît souvent incongru à l'historien : cette incertitude méthodologique autant que narrative questionne le statut même de l'enquête historique. L'analyse contrefactuelle ne doit pourtant pas être considérée comme une forme de menace ou d'hétérodoxie pour la science historique, qu'elle peut enrichir au contraire par la stricte recherche de la causalité autant que par l'exploration des possibles : le possible demeure une des catégories «les plus difficiles à penser en histoire, pour qui ne se résout pas à user des commodités de la fiction ${ }^{71} »$, mais aussi l'un des enjeux majeurs d'une réflexion sur l'intelligibilité des sociétés passées. Face à ce défi épistémologique, un effort d'imagination est nécessaire.

69. Q. Deluermoz et P. Singaravélou, «Explorer...», art. cit.

70. Parmi d'autres, voir Jean-Yves Grenier, Claude Grignon et Pierre-Michel Menger (dir.), Le Modèle et le récit, Paris, Éditions de la Maison des sciences de l'homme, 2001.

71. Jacques Revel, «Ressources narratives et connaissance historique», Enquête, 1 (1995) : Les terrains de l'enquête, p. 43-70. URL : http://enquete.revues.org/document262.html. 